Band 26/Vol. 26, 1999

Gegründet 1973 als «Die Infusionstherapie» durch H. Reissigl, H. Grobecker, U. Henneberg, K.H. Bäßler

Bisherige Herausgeber und Schriftleiter:

H.-D. Viering (1973-1977), A. Grünert (1982-1992),

G. Kleinberger (1982-1988), K. Widhalm (1988-1992),

G. Wolfram (1990-1992), J. Eckart (1992-1997),

H. Forst (1992-1997), K. Meßmer (1992-1997),

K. Peter (1992-1997), K. H. Usadel (1992-1997),

B. Zwißler (1995-1997)
Offizielles Organ der Deutschen Gesellschaft für

Transfusionsmedizin und Immunhämatologie

\section{Verantwortlicher Herausgeber}

V. Kretschmer, Marburg

\section{Herausgeber}

W. Dzik, Boston, MA

H.G. Klein, Bethesda, MD

W. Sibrowski, Münster

W. Stangel, Hannover

\section{Schriftleitung}

K. Gutensohn, Hamburg

A. Sputtek, Hamburg

\title{
Ressort-Herausgeber
}

Blutprodukte

M. Böck, Würzburg

G. Holzberger, Kassel

H. Klüter, Mannheim

Klinische Transfusionsmedizin

M.U. Heim, Magdeburg

A.G. Salama, Berlin

Transplantation

G. Bein, Gießen

M. Wiesneth, Ulm

Immunhämatologie

V. Kiefel, Rostock

H.H. Sonneborn, Dreieich

Hämostaseologie

A. Greinacher, Greifswald

M. Köhler, Göttingen

E. Seifried, Frankfurt/M.
Sicherheit, Risiken und

Nebenwirkungen

G. Caspari, Gießen

U.T. Seyfert, Homburg/Saar

Rechtsfragen, Standards

E. Biermann, Nürnberg

R. Dörner, Köln

R. Seitz, Langen

Autologe Hämotherapie, blutsparende Verfahren

J. Biscoping, Karlsruhe

W. Mempel, München

G. Singbartl, Hamburg

\section{Wissenschaftlicher Beirat}

J.-F. Baron, Paris

B. Blauhut, Linz

H. Borberg, Köln

U.B. Brückner, Ulm

W. Dahr, Bergisch Gladbach

H.W. Doerr, Frankfurt/M.

R. Eckstein, Erlangen

H. Forst, Augsburg

L. Frey, München

W. Gerlich, Gießen

S. Goldmann, Ulm

E. Götz, Darmstadt

H. Gombotz, Graz

H. Grosse-Wilde, Essen

L. Gürtler, Greifswald

P. Hanfland, Bonn
E. Hansen, Regensburg

P. Höcker, Wien

J.P. Isbister, Sydney

H. Kiesewetter, Berlin

H.-J. Kolb, München

B. Kubanek, Ulm

P. Kühnl, Hamburg

H. Laubenthal, Bochum

K. Meßmer, München

N. Müller, Essen

G. Müller-Berghaus, Bad Nauheim

E. Northoff, Tübingen

U.E. Nydegger, Bern

K. Peter, München
R. Pietersz, Amsterdam

G.F. Riedler, Luzern

D. Roelcke, Heidelberg

G. Schmitz, Regensburg

N. Schmitz, Kiel

D. Schönitzer, Innsbruck

W. Schramm, München

D. Spahn, Zürich

L. Sunder-Plassmann, Ulm

D. Voak, Cambridge, UK

E. Wenzel, Homburg/Saar

K. Werdan, Halle/Saale

R. Zander, Mainz

B. Zwißler, München 


\section{Bibliographische Dienste}

Biological Abstracts

Current Contents/Clinical Medicine

Excerpta Medica/EMBASE

Medical Documentation Service

Reference Update

Research Alert

Science Citation Index

SCISEARCH Database

S. Karger $\cdot$ Medical and Scientific Publishers $\cdot$ Basel $\cdot$ Freiburg $\cdot$ Paris $\cdot$ London $\cdot$ New York $\cdot$ New Delhi $\cdot$ Bangkok $\cdot$ Singapore $\cdot$ Tokyo $\cdot$ Sydney

Die Zeitschrift erscheint zweimonatlich; pro Jahr erscheint 1 Band zu je 6 Heften. DGTI-Mitglieder erhalten die Zeitschrift im Rahmen ihrer Mitgliedschaft. Bezugspreis für Jahrgang 26, 1999, DEM 198,- / CHF 154,-, einschließlich MWSt., zuzüglich Postgebühren. Der Abonnementpreis ist im voraus zahlbar. Das Abonnement der Zeitschrift läuft weiter, wenn es nicht spätestens 4 Wochen vor Abschluß eines Bandes abbestellt wird. Abonnementbestellungen können bei jeder Buchhandlung oder direkt beim Verlag aufgegeben werden:

Deutschland:

S. Karger GmbH

Lörracher Str. 16a

D-79115 Freiburg

Telefon +49761452070

E-mail Information@Karger.de

Postbank München

Postbank Mün
614 40-808
Anzeigen: S. Karger

Verlag für Medizin und Naturwissenschaften $\mathrm{GmbH}$

Lörracher Str. 16a, D-79115 Freiburg,

Telefon +49761452070

Gültig ist die Preisliste Nr. 14 vom 1. Januar 1999.

Für den Inhalt außerhalb des redaktionellen Teiles (insbesondere Anzeigen, Industrieinformationen, Pressezitate un Kongreßinformationen) übernehmen Schriftleitung, Beirat und Verlag keine Gewähr.

Eine Markenbezeichnung kann warenzeichenrechtlich geschützt sein, auch wenn bei ihrer Verwendung in dieser Zeitschrift das Zeichen (®) oder ein anderer Hinweis auf etwa bestehende Schutzrechte fehlen sollte. Für Satzfehler, insbesondere bei Dosierungsangaben, wird keine Gewähr übernommen. Die Zeitschrift sowie alle in ihr enthaltenen einzelnen Beiträge und Abbild sowie alle in ihr enthate wertung, die nicht ausdrücklich vom Urheberrechtsgesetz zugelassen ist, bedarf der vorherigen Zustimmung des Verlags. Das gilt insbesondere für Vervielfältigungen, Bearbeitungen, Über-
setzungen, Mikroverfilmungen und die Einspeicherung und setzungen, Mikroverfilmungen und die
Verarbeitung in elektronischen Systemen. (c) Copyright 1999 by S. Karger

Verlag für Medizin und Naturwissenschaften $\mathrm{GmbH}$ Lörracher Str. 16a, D-79115 Freiburg

Verlagsleitung und presserechtlich verantwortlich: Sibylle Hopf Produktionsleitung: Georg Brunner Anzeigenleitung: Susanne Meister

Herstellung: Konkordia Druck GmbH, Eisenbahnstraße 31, D-77815 Bühl

\section{KARGER}

(c) 1999 S. Karger GmbH, Freiburg

Fax +49 7614520714 


\section{Editorials}

15 Genotypisierung und Phänotypisierung: Zwei Seiten einer Medaille

Northoff, H. (Tübingen); Flegel, W.A. (Ulm)

5258 Neue Entwicklungen in der Blutgruppenserologie Voak, D. (Cambridge, UK)

Klinische Information

6321 Humane T-Zell-lymphotrope Viren Typ 1 und 2 (HTLV-I/-II)

Arbeitskreis Blut, Untergruppe «Bewertung Blutassoziierter Krankheitserreger»

Übersichtsarbeiten

17 Aspekte der Prophylaxe von thrombotischen Embolien bei Operationen

Bergqvist, D. (Uppsala)

114 Zusammenhang zwischen AB0-Blutgruppe und Thromboseneigung. Was ist gesichert?

Tonn, T.; Seifried, E. (Frankfurt/M.)

120 Blutplättchen sind zirkulierende Speicher für Adhäsionsproteine, Entzündungsmediatoren und Immunglobuline - Bedeutung bei nonhämolytischen Transfusionsreaktionen Klinger, M.H.F.; Klüter, H. (Lübeck)

278 Aspekte der Qualitätssicherung im Rahmen der peripheren Blutstammzelltransplantation Cassens, U.; Ahlke, C.; Sibrowski, W. (Münster)

285 Zytokin-induzierte Mobilisation peripherer Blutstammzellen aus dem Steady-state bei Patienten mit soliden Tumoren und hämatologischen Neoplasien Kröger, N.; Zeller, W.; Hassan, H.T.; Krüger, W.; Renges, H.; Rüssmann, B.; Fuchs, N.; Rauhöft, C.; Gutensohn, K.; Löliger, C.; Erttmann, R.; Kühnl, P.; Zander, A.R. (Hamburg)

292 Stammzellmobilisation bei gesunden Spendern: Derzeitiger Stand

Dreger, P.; Schmitz, N. (Kiel)

296 Nachweis disseminierter epithelialer Tumorzellen: Methoden und klinische Anwendung Pantel, K.; Witter, K. (München)
3130 Zellselektion im Knochenmark und Stammzelltransplantation

Krüger, W.; Kröger, N.; Gutensohn, K.; Tögel, F.; Zander, A.R. (Hamburg)

3140 Kombinierte positive/negative Selektion bei der Stammzelltransplantation: Perspektiven der Stammzellmanipulation

Mapara, M.Y.; Körner, I.J.; Lentzsch, S.; Hildebrandt, M.; Dörken, B. (Berlin)

3146 Fluoreszenz-in-situ-Hybridisierung (FISH): Eine wirksame Methode zur Bestimmung von genetischen Anomalitäten während der Metaphase und in Interphase-Nuclei Dierlamm, J. (Hamburg); Wlodarska, I.; Michaux, L. (Leuven); Stefanova, M.; Seeger, D.; Hinz, K. (Hamburg); Hagemeijer, A. (Leuven); Hossfeld, D.K. (Hamburg)

3152 Granulozytopenien und Transfusionsreaktionen infolge granulozytärer Antikörper Bux, J. (Gießen)

4186 Optimaler Hämatokrit 30\%: Abschied von einer Illusion Zander, R. (Mainz)

4193 Die Rolle des Monozytenoberflächenantigens CD14 und des Lipopolysaccharid-bindenden Proteins in der Erkennung von Endotoxin und gramnegativen Bakterien: In-vitro-Funktion, tierexperimentelle Modelle und therapeutische Ausblicke Stelter, F. (Greifswald)

4202 Monoklonale Antikörper und Blutgruppenbestimmung sowie weiterführende Technologien Sonneborn, H.-H. (Dreieich); Dahr, W. (Bergisch-Gladbach); Helmbold, W. (Meckesheim); Kasulke, D. (Baden-Baden)

\section{Entwicklungsstrategien zur Prävention}

Kathetermaterial-assoziierter Thrombosen und Infektionen

Schierholz, J.M. (Köln); Seyfert, U.T. (Homburg/Saar); Rump, A.F.E (Aachen); Beuth, J.; Pulverer, G. (Köln)

Originalarbeiten

126 Schutz gelagerter Erythrozyten gegen oxidativen Streß und unzureichende physiologische Funktion durch Enantiomere der $\alpha$-Liponsäure Geise, W.; Conrad, F.; Henrich, H.A. (Würzburg)

\section{KARGER}

(c) 1999 S. Karger GmbH, Freiburg

Fax +497614520714

E-mail kargergmbh@aol.com

www.karger.con 
134 Antithrombozytäre Antikörper und Fieber: Deren Zusammenhang bei polytransfundierten Patienten mit hämato-onkologischen Erkrankungen

Kurz, M.; Eichelberger, B.; Greinix, H.; Höcker, P.; Kahls, P.; Knöbl, P.; Mayr, W.R.; Panzer, S. (Wien)

138 Untersuchungen zur Spezifität der drei ScreeningParameter HBsAg, Anti-HIV-1/HIV-2 und Anti-HCV mit dem neuen ABBOTT PRISM ${ }^{\circledR}$-System im Vergleich zum ABBOTT COMMANDER ${ }^{\circledR}$-System Weichert, W.; Bialleck, H.; Kirchmaier, C.; Roth, W.; Seifried, E. (Frankfurt/M.)

145 Aufnahme von Fluorescein-markierter Hydroxyäthylstärke durch organgebundene und zirkulierende Zellen des retikuloendothelialen Systems Förster, H.; Meier, B.; Asskali, F. (Frankfurt/M.)

153 Eine durchflußzytometrische Methode zum Nachweis von Leukozytenkontaminationen in Leukozytendepletierten Blutprodukten Andea, A.; Garritsen, H.S.P.; Cassens, U.; Kelsch, R.; Sibrowski, W. (Münster)

157 Thoraxbioimpedanz während Periduralanästhesie Wirkung unterschiedlicher HydroxyethylstärkeLösungen auf sympathikolytisch induzierte Schlagvolumenänderungen Heine, J.; Bund, M.; Piepenbrock, S. (Hannover)

2103 Bedeutung der Vorläuferzellwerte des peripheren Blutes der Ruhehämatopoese für die Abschätzung der Anzahl an Blutvorläuferzellen, die nach einer Granulozyten-koloniestimulierender-Faktorunterstützten Chemotherapie mobilisiert werden Fruehauf, S.; Schmitt, K.; Veldwijk, M.R.; Schiedlmeier, B.; Martin, S.; Zeller, W.J.; Haas, R. (Heidelberg)

2110 Das Mannheimer Nabelschnurblut-Projekt: Erfahrungen zur Sammlung und Aufarbeitung der ersten 880 unverwandten Transplantate aus Plazentablut Eichler, H.; Richter, E.; Leveringhaus, A.; Zieger, W.; Watz, E.; Friedmann, G.; Kerowgan, M.; Goldmann, S.F. (Mannheim)

2115 Phänotyp und Funktion antigenpräsentierender dendritischer Zellen, die aus Monozyten des peripheren Bluts generiert wurden Bauer, Y.; Jäger, C.; Kramer, M.D.; Wallich, R. (Heidelberg)

3158 Einflußgrößen bei der Bestimmung von CD34+ Zellen im Blut und in Leukapherisaten

Cassens, U.; Garritsen, H. (Münster); Gutensohn, K. (Hamburg); Sibrowski, W. (Münster)

3168 Sammlung von peripheren Progenitorzellen bei Kindern mit reduzierter Produktvolumentechnik Moog, R.; Basu, O.; Fuchs, N.; Kremens, B.; Müller, N. (Essen)
3172 Präzise Quantifizierung von CD34+ Zellen mittels multiparametrischer Durchflußzytometrie und fluoreszierender Mikropartikel

Schlenke, P.; Frohn, C.; Müller-Steinhardt, M.; Hammers, H.-J.; Saballus, M.; Klüter, H. (Lübeck)

4212 Einfluß von 30-Gy-Gamma-Bestrahlung auf die Qualität von Erythrozytenkonzentraten in verschiedenen Lagerungsmedien Bäumler, H.; Radtke, H.; Haas, T.; Latza, R.; Kiesewetter, H. (Berlin)

4222 Leukozytenzählung mit dem Nageotte-Hämocytometer: Anwendung der Fluoreszenzmikroskopie Graldi, G.; Matteucci, G.; Reverberi, R. (Ferrara)

4226 Diätische Beeinflussung der bakteriellen Clearance und RES-Phagozytosekapazität durch parenterale Applikation von Omega-3-Fettsäuren Kelbel, I. (Ulm); Koch, T. (Dresden); Prechtl, A. (Gießen); Heller, A. (Dresden); Schlotzer, E. (Bad Homburg); Schiefer, H.G.; Neuhof, H. (Gießen)

4234 Alloantikörper gegen hochfrequente Erythrozytenantigene Heuft, H.-G.; Genth, R.; Wittmann, G.; Salama, A. (Berlin)

4240 Kälteagglutinin-Isotypen Kreft, H.; Hack, H.; Roelcke, D. (Heidelberg)

4244 Eine neue Festphasenmethode zur AB0Blutgruppenbestimmung, Rhesus-Phänotypisierung und Kell-Bestimmung Uthemann, H.; Prager-Eberle, M.; Sturmfels, L.; Lenhard, V. (Dreieich)

5262 Photoinaktivierung von frischgefrorenem Plasma mit Methylenblau: Wirkung auf HIV, HCV und Parvovirus B19

Iudicone, P.; Andreoni, M.; Lavorino, C.; Martorana, M.-C.; Nicastri, E. (Roma); Azzi, A. (Firenze); Quintiliani, L. (Roma)

5267 Entnahmereaktionen und Straßenverkehrsrisiken bei der Eigenblutentnahme

Felleiter, P. (Wien); Freudenberg, J.; Wiesneth, M.; Kubanek, B. (Ulm)

5272 Nebenwirkungen bei präoperativer Eigenblutspende und Plasmapherese - Eine 6-Jahres-Analyse bei 28244 Patienten mit 50542 autologen Spenden Singbartl, G. (Hamburg); Schleinzer, W. (Heilbronn)

5288 In-line-Leukozytendepletion von ThrombozytaphereseKonzentraten

Zeiler, T.; Kretschmer, V. (Marburg)

5293 Effekt von Vorspendenergebnissen und vertraulichem Selbstausschluß auf Prävalenz und Inzidenz von HCV bei Blutspendern des DRK-Blutspendedienstes Nordrhein-Westfalen

Caspari, G. (Gießen); Fiedler, H.; Hornstein, C.; Muß, P. (Hagen); Gerlich, W.H. (Gießen); Lefèvre, H. (Hagen) 
6328 Nachweis rarer Fälle von HTLV-I- und -II-Infektionen und eine hohe Anzahl HTLV-seroindeterminanter Ergebnisse bei bayrischen Blutspendern

Fleischer, C.; Kücherer, C. (Berlin); Michel, P.; Weise, W. (München); Stahl-Hennig, C.; Bodemer, C.; Hunsmann, G. (Göttingen); Pauli, G. (Berlin)

6335 Risiko der HIV-, HCV- und HBV-Übertragung durch Blutpräparate. Aktuelle Daten 1997 und 1998 aus der Studie zur Epidemiologie von HIV und Hepatitisinfektionen bei Blutspendern in Deutschland Glück, D. (Ulm)

6339 Evaluierung von proinflammatorischen Zytokinen in vor der Lagerung leukozytendepletiertem Vollblut mit Hilfe von effizienter Inline-Filter

Bontadini, A.; Fruet, F.; Ruscitto, M.C.; Manfroi, S.; Conte, R. (Bologna)

6344 Thrombozytenfunktion und Parameter der hämostatischen Aktivierung in Plasmaspendern Vuk, T.; Maglov, C.; Godec, D.; Tomicic, M.; Balija, M.; Grgicevic, D. (Zagreb)

6348 Die Prävalenzrate von Erythrozytenantikörpern während der Schwangerschaft im Bundesland Salzburg. Ein Vergleich zwischen D-positiven und D-negativen Frauen Mühlbacher, A.; Michl, U. (Salzburg)

6353 Initiale Hämoglobinkonzentration und Spendeplanung als Einflußfaktoren der Erythropoese bei der Eigenblutspende

Gesemann, M.; Genter, P.R.; Scheiermann, N. (Frankfurt/M.)

6360 Erstellung von Blutvolumenreferenzwerten für erythrozytenmarkierende Indikatorverdünnungsmethoden anhand von Literaturdaten Lauermann, I.; Hecker, H.; Kirchner, E. (Hannover)

Qualitätsmanagement

162 Zählung geringer Mengen von Leukozyten in leukoreduzierten Blutkomponenten Dzik, S. (Boston)

5300 Veränderung der Spenderausschlußrate bei der Gewinnung von Apheresespendern durch die Neufassung der «Richtlinien zur Blutgruppenbestimmung und Bluttransfusion (Hämotherapie)» Glaser, A.; Zimmermann, R.; Zingsem, J.; Weisbach, V.; Neidhardt, B.; Eckstein, R. (Erlangen)

\section{Kurzmitteilung}

5306 Bestimmung von spender- und produktbezogenen Parametern bei der Plasmapherese Kießig, S.T.; Saavedra-Maldonado, C. (Heidelberg); Aicher, H. (Wien); Storch, H. (Heidelberg)

Briefe an die Herausgeber

2119

Gesellschaftsmitteilungen

1 66, 4247, 6367

Industrieforum

1 68, 3 179, 4 249, 5310

Tagungen und Kongresse

1 69, 2 122, $3180,4250,5312,6369$

Sonderheft 1

32. Jahreskongreß der Deutschen Gesellschaft für Transfusionsmedizin und Immunhämatologie (DGTI) Münster-Westfalen, 5.-8. Oktober 1999. Abstracts Hrsg.: Sibrowski W. (Münster); Kleesiek K. (Bad Oeyenhausen)

\section{Sonderheft 2}

31. Jahreskongreß der Deutschen Gesellschaft für Transfusionsmedizin und Immunhämatologie (DGTI) und Herbst-Symposium '98 der Schweizerischen Vereinigung für Transfusionsmedizin (SVTM)

Bern, 6.-9. Oktober 1998. Selected Contributions Hrsg.: Nydegger U.E. (Bern); Riedler G.F. (Luzern); Kretschmer V. (Marburg)

6371 Autorenverzeichnis 1999

6373 Sachwortverzeichnis 1999 
Editorials

15 Genotyping and Phenotyping: The Two Sides of One Coin

Northoff, H. (Tübingen); Flegel, W.A. (Ulm)

5258 New Developments in Blood Group Serology

Voak, D. (Cambridge, UK)

Clinical Information

6321 Human T-Cell Lymphotropic Viruses Type 1 and 2 (HTLV-I/-II)

Arbeitskreis Blut, Untergruppe «Bewertung Blutassoziierter Krankheitserreger»

Review Articles

17 Aspects on Prophylaxis of Thromboembolism in Surgery Bergqvist, D. (Uppsala)

114 AB0 Blood Group and Individual Risk for Thrombosis. The Bottom Line

Tonn, T.; Seifried, E. (Frankfurt/M.)

120 Blood Platelets Are Circulating Stores for Adhesive Proteins, Inflammatory Mediators, and Immunoglobulins - Role in Nonhemolytic Transfusion Reactions

Klinger, M.H.F.; Klüter, H. (Lübeck)

278 Aspects of Quality Assurance in the Preparation of Peripheral Blood Stem Cells for Transplantation Cassens, U.; Ahlke, C.; Sibrowski, W. (Münster)

285 Steady-State Mobilization of Peripheral Blood Progenitor Cells by Cytokines Alone in Patients with Solid Tumors or Hematological Malignancies Kröger, N.; Zeller, W.; Hassan, H.T.; Krüger, W.; Renges, H.; Rüssmann, B.; Fuchs, N.; Rauhöft, C.; Gutensohn, K.; Löliger, C.; Erttmann, R.; Kühnl, P.; Zander, A.R. (Hamburg)

292 Stem Cell Mobilization in Healthy Donors: Current Status

Dreger, P.; Schmitz, N. (Kiel)

296 Detection of Disseminated Epithelial Tumor Cells: Methods and Clinical Implications Pantel, K.; Witter, K. (München)
3130 Cell Selection in Marrow and Stem Cell Transplantation Krüger, W.; Kröger, N.; Gutensohn, K.; Tögel, F.; Zander, A.R. (Hamburg)

3140 Combined Positive/Negative Selection in Stem Cell Transplantation: Perspectives in Graft Engineering Mapara, M.Y.; Körner, I.J.; Lentzsch, S.; Hildebrandt, M.; Dörken, B. (Berlin)

3146 Fluorescence in situ Hybridization (FISH): A Powerful Method for the Detection of Genetic Abnormalities in Metaphases and Interphase Nuclei

Dierlamm, J. (Hamburg); Wlodarska, I.; Michaux, L. (Leuven); Stefanova, M.; Seeger, D.; Hinz, K. (Hamburg); Hagemeijer, A. (Leuven); Hossfeld, D.K. (Hamburg)

3152 Granulocyte Antibody-Mediated Neutropenias and Transfusion Reactions Bux, J. (Gießen)

4186 Optimal Hematocrit 30\%: Farewell to a Fallacy Zander, R. (Mainz)

4193 Role of the Monocyte Differentiation Antigen CD14 and Lipopolysaccharide-Binding Protein in the Recognition of Endotoxin and Gram-Negative Bacteria: In vitro Function, Animal Models, and Therapeutic Implications Stelter, F. (Greifswald)

4202 Monoclonal Antibodies and Blood Group Determination as well as Further Technologies Sonneborn, H.-H. (Dreieich); Dahr, W. (Bergisch-Gladbach); Helmbold, W. (Meckesheim); Kasulke, D. (Baden-Baden)

5278 Strategies for the Prevention of Catheter MaterialAssociated Thrombosis and Bloodstream Infections Schierholz, J.M. (Köln); Seyfert, U.T. (Homburg/Saar); Rump, A.F.E. (Aachen); Beuth, J.; Pulverer, G. (Köln)

Original Articles

126 Protection of Stored Erythrocytes against Oxidative Stress and Insufficient Physiological Function by Enantiomers of $\alpha$-Lipoic Acid Geise, W.; Conrad, F.; Henrich, H.A. (Würzburg)

134 Platelet Antibodies and Fever: Their Association in Multitransfused Patients with Hemato-Oncological Diseases

Kurz, M.; Eichelberger, B.; Greinix, H.; Höcker, P.; Kahls, P.; Knöbl, P.; Mayr, W.R.; Panzer, S. (Wien) 
138 Specificity of Screening Assays for HBsAg, Anti-HIV-1/HIV-2 and Anti-HCV on the Newly Developed ABBOTT PRISM ${ }^{\circledR}$ System Weichert, W.; Bialleck, H.; Kirchmaier, C.; Roth, W.; Seifried, E. (Frankfurt/M.)

145 Ingestion of Fluorescein-Labeled Hydroxyethyl Starch by Organ-Bound and Circulating Cells of the Reticuloendothelial System Förster, H.; Meier, B.; Asskali, F. (Frankfurt/M.)

153 A Flow-Cytometric Method for Assessing Leukocyte Contamination in White-Cell-Depleted Blood Products Andea, A.; Garritsen, H.S.P.; Cassens, U.; Kelsch, R.; Sibrowski, W. (Münster)

157 Thoracic Bioimpedance During Extradural Anesthesia - Effect of Different Hydroxyethyl Starch Solutions on Stroke Volume under Sympatholysis Heine, J.; Bund, M.; Piepenbrock, S. (Hannover)

2103 Relevance of Peripheral Blood Progenitor Cell Counts during Steady-State Hematopoiesis to Estimate the Number of Progenitor Cells Mobilized following Granulocyte Colony-Stimulating Factor-Supported Chemotherapy

Fruehauf, S.; Schmitt, K.; Veldwijk, M.R.; Schiedlmeier, B.; Martin, S.; Zeller, W.J.; Haas, R. (Heidelberg)

2110 The Mannheim Cord Blood Project: Experience in Collection and Processing of the First 880 Banked Unrelated Cord Blood Transplants

Eichler, H.; Richter, E.; Leveringhaus, A.; Zieger, W.; Watz, E.; Friedmann, G.; Kerowgan, M.; Goldmann, S.F. (Mannheim)

2115 Phenotype and Function of Antigen-Presenting Dendritic Cells Generated from Peripheral Blood Monocytes Bauer, Y.; Jäger, C.; Kramer, M.D.; Wallich, R. (Heidelberg)

3158 Factors Affecting the Determination of CD34+ Cells in Blood and Leukapheresis Products

Cassens, U.; Garritsen, H. (Münster); Gutensohn, K. (Hamburg); Sibrowski, W. (Münster)

3168 Collection of Peripheral Progenitor Cells in Pediatric Patients with Reduced Volume Technique Moog, R.; Basu, O.; Fuchs, N.; Kremens, B.; Müller, N. (Essen)

3172 Precise CD34+ Quantification Using a MultiParameter Flow-Cytometric Method with Fluorescent Microparticles

Schlenke, P.; Frohn, C.; Müller-Steinhardt, M.; Hammers, H.-J.; Saballus, M.; Klüter, H. (Lübeck)

4212 Influence of 30 Gy Gamma Irradiation on the Quality of Red Blood Cell Concentrates in Several Storage Media Bäumler, H.; Radtke, H.; Haas, T.; Latza, R.; Kiesewetter, H. (Berlin)
4222 Leukocyte Counting with the Nageotte Hemocytometer: Use of Fluorescence Microscopy Graldi, G.; Matteucci, G.; Reverberi, R. (Ferrara)

4226 Effects of Parenteral Application of Fish Oil versus Soy Oil Emulsions on Bacterial Clearance Functions Kelbel, I. (Ulm); Koch, T. (Dresden); Prechtl, A. (Gießen); Heller, A. (Dresden); Schlotzer, E. (Bad Homburg); Schiefer, H.G.; Neuhof, H. (Gießen)

4234 Alloantibodies Directed against High-Frequency Red Blood Cell Antigens

Heuft, H.-G.; Genth, R.; Wittmann, G.; Salama, A. (Berlin)

4240 Cold Agglutinin Isotypes

Kreft, H.; Hack, H.; Roelcke, D. (Heidelberg)

4244 A New Solid-Phase Method for ABO Grouping, Rh Phenotyping and Kell Determination Uthemann, H.; Prager-Eberle, M.; Sturmfels, L.; Lenhard, V. (Dreieich)

5262 Photodynamic Treatment of Fresh Frozen Plasma by Methylene Blue: Effect on HIV, HCV, and Parvovirus B19

Iudicone, P.; Andreoni, M.; Lavorino, C.; Martorana, M.-C.; Nicastri, E. (Roma); Azzi, A. (Firenze); Quintiliani, L. (Roma)

5267 Donor Reactions and Traffic Risks in Autologous Blood Predeposits Felleiter, P. (Wien); Freudenberg, J.; Wiesneth, M.; Kubanek, B. (Ulm)

5272 Adverse Events in Autologous Blood Donation and Plasmapheresis - a 6-Year Analysis in 28,244 Patients with 50,542 Donations Singbartl, G. (Hamburg); Schleinzer, W. (Heilbronn)

5288 In-Line Leukocyte Depletion of Thrombocyte Apheresis Concentrates Zeiler, T.; Kretschmer, V. (Marburg)

5293 Effect of Donor Selection and Confidential SelfExclusion on Prevalence and Incidence of $\mathrm{HCV}$ in Blood Donors at the Red Cross Blood Transfusion Service of North Rhine-Westphalia (Germany) Caspari, G. (Gießen); Fiedler, H.; Hornstein, C.; Muß, P. (Hagen); Gerlich, W.H. (Gießen); Lefèvre, H. (Hagen)

6328 Detection of Rare Cases of HTLV-I and -II Infections and High Numbers of HTLV-Seroindeterminate Results in Bavarian Blood Donors

Fleischer, C.; Kücherer, C. (Berlin); Michel, P.; Weise, W. (München); Stahl-Hennig, C.; Bodemer, C.; Hunsmann, G. (Göttingen); Pauli, G. (Berlin) 
6335 Risk of HIV, HCV and HBV Transmission by Blood Products. Current Data 1997 and 1998 - Study on the Epidemiology of HIV and Hepatitis in Blood Donors in Germany

Glück, D. (Ulm)

6339 Evaluation of Proinflammatory Cytokines in Prestorage Leukodepleted Whole Blood Using a HighPerformance Inline Filter

Bontadini, A.; Fruet, F.; Ruscitto, M.C.; Manfroi, S.; Conte, R.

(Bologna)

6344 Platelet Function and Markers of Hemostatic

Activation in Plasma Donors

Vuk, T.; Maglov, C.; Godec, D.; Tomicic, M.; Balija, M.; Grgicevic, D. (Zagreb)

6348 Prevalence Rate of Red Blood Cell Antibodies during Gravidity in the Federal Province of Salzburg. A Comparison between D-Positive and D-Negative Women

Mühlbacher, A.; Michl, U. (Salzburg)

6353 Association of Erythropoiesis during Autologous

Blood Donation with Initial Hemoglobin Concentration and Length of Donation Period

Gesemann, M.; Genter, P.R.; Scheiermann, N. (Frankfurt/M.)

6360 Elaboration of Reference Values for Blood Volume Determination Methods with Red Blood Cell Labelling Indicator Dilution Techniques Using Values from Literature

Lauermann, I.; Hecker, H.; Kirchner, E. (Hannover)

Quality Management

162 Counting Low Numbers of Leukocytes in Leukoreduced Blood Components Dzik, S. (Boston)

5300 Change of the Deferral Rate of Volunteer Apheresis Donors by the New Edition of the 'German Guidelines for Blood Grouping and Blood Transfusion

(Haemotherapy)'

Glaser, A.; Zimmermann, R.; Zingsem, J.; Weisbach, V.; Neidhardt, B.; Eckstein, R. (Erlangen)
Short Communication

5306 Detection of Donor- and Product-Specific Parameters in Plasmapheresis

Kießig, S.T.; Saavedra-Maldonado, C. (Heidelberg);

Aicher, H. (Wien); Storch, H. (Heidelberg)

Letters to the Editors

2119

Society Bulletins

1 66, 4247,6367

Industrial Forum

$\mathbf{1} 68, \mathbf{3} 179, \mathbf{4} 249, \mathbf{5} 310$

Meetings and Conferences

$\mathbf{1} 69, \mathbf{2} 122, \mathbf{3} 180, \mathbf{4} 250, \mathbf{5} 312, \boldsymbol{6} 369$

Special Issue 1

32. Jahreskongreß der Deutschen Gesellschaft für Transfusionsmedizin und Immunhämatologie (DGTI) Münster-Westfalen, 5.-8. Oktober 1999. Abstracts Hrsg.: Sibrowski W. (Münster); Kleesiek K. (Bad Oeyenhausen)

Special Issue 2

31. Jahreskongreß der Deutschen Gesellschaft für Transfusionsmedizin und Immunhämatologie (DGTI) und Herbst-Symposium '98 der Schweizerischen Vereinigung für Transfusionsmedizin (SVTM)

Bern, 6.-9. Oktober 1998. Selected Contributions

Hrsg.: Nydegger U.E. (Bern); Riedler G.F. (Luzern); Kretschmer V. (Marburg)

6371 Author Index 1999

6375 Subject Index 1999 